\begin{tabular}{|l|c|c|c|}
\hline $\begin{array}{l}\text { ACTA CLASSICA } \\
\text { UNIV. SCIENT. DEBRECEN. }\end{array}$ & LIII. & 2017. & \\
\hline
\end{tabular}

\title{
JUNGFRAUEN IN WAFFEN: CAMILLA VIRGO, IUTURNA VIRAGO ${ }^{1}$
}

\author{
VON THOMAS KÖVES-ZULAUF
}

\begin{abstract}
The paper starts with the analysis of the ambiguous figure of the "Italic Amazon" Camilla in the Aeneis. Two main factors are analysed, the role of the spear in the life of this "armed virgin" and her relations with natural surroundings, especially with rivers like the Amasenus and with lakes and marshes generally. Both prove to be fateful. The second factor is also noticeable with the other armed virgin of the Aeneis, Iuturna, who emerges as an upgraded and contrasting parallel to Camilla. Iuturna virago shows similarities with another famous virago of Latin literature, the Paluda virago of Ennius, and this gives an opportunity to evoke other women of the Roman tradition in connection with rivers and marshes, a phenomenon which proves to be a suitable topic for future exanimation.
\end{abstract}

Keywords: Camilla, Iuturna, Paluda virago, Caprae palus. Amasenus, Metabus

A

Camilla

I

Horrenda virgo-decus Italiae

In der Aeneis Vergils tritt in einem entscheidenden Moment der Kriegsereignisse die Jungfrau Camilla auf, entschlossen - das Kriegsmonopol der Männer usurpierend - am Verteidigungskampf der Italiker gegen die Fremden teilzunehmen, die eine neue Heimat in Italien zu erkämpfen sich anschicken. Der Führer der Italiker Turnus starrt das kriegsbereite Mädchen an, das ihm als eine „horrenda virgo“, als eine „schreckliche Jungfrau“(XI 507) ${ }^{2}$ erscheint. Die

\footnotetext{
${ }^{1}$ Überarbeiteter Text eines Vortrags, gehalten in der Gesellschaft für Altertumswissenschaften aus Anlaß der Verleihung der Ábel Jenő - Medaille 2017 in Budapest.

${ }^{2}$ Gelegentlich wird diese Charakterisierung Camillas als „horrenda“ mit „Ehrfurcht gebietend" (Binder z.St.),"bewundernswert" (Georges 1962 (1913)) s.v.) übersetzt d.h. als positive Eigenschaft und dadurch der Widerspruch zum folgenden Lob beseitigt. Aber hier kann horrenda keine positive Eigenschaft bezeichnen, wie alle Parallelstellen in der Aeneis es beweisen. Nicht-
} 
Sprache verschlägt ihm dieser Anblick aber nicht. Im Gegenteil: er spricht sie mit feierlichen Worten an. Seine Worte sind sehr überraschend. Er apostrophiert die Jungfrau, die ihm als Schrecken erregend erscheint, als ,Zierde Italiens": $O$ decus Italiae virgo, $=$ Oh Zierde Italiens Jungfrau (XI 508), lautet seine Anrede. Diese Qualifizierung ist umso überraschender, da sonst als Stolz Italiens im Rahmen einer Lobpreisung Italiens regelmäßig nur Männer vorkom$\mathrm{men}^{3}$. Wie kann eine Jungfrau zugleich als Schreckgestalt und als Zierde qualifiziert werden? Eine Antwort auf diesen Widerspruch zu suchen ist der Zweck meines Vortrags.

Ich rufe die Lebensgeschichte Camillas kurz in Erinnerung. Der Herrscher der Stadt Privernum wird von seinen Untertanen vertrieben. Der Flüchtende nimmt sein einige Wochen altes Kind Camilla mit. Als ein von Sturm aufgewühlter Strom ihm den Weg versperrt, bindet er den Säugling an seine Lanze, weiht es der Göttin Diana unter der Bedingung, daß sie für die Errettung Camillas sorgt und wirft es mit Erfolg über den Strom, bevor er selber hinüberschwimmt. Von nun an lebt das Kind mit seinem Vater in der Wildnis ein Hirtenleben, sein Leben im Dienste Dianas der Jagd und der Bewahrung der Unberührtheit seines jungfräulichen Körpers widmend. Eines Tages wird sie aber ihrer dianischen Lebensart untreu, gibt der Sehnsucht nach, die unter der Führung des Aeneas in Italien sich ansiedeln wollenden Troianer zum Kampf zu reizen. ${ }^{4}$ So kämpft sie eine Zeit lang als italische Amazone mit Erfolg gegen die eindringenden Männer und ihre etruskischen Verbündeten, bis am Ende ein etruskischer Priester Arruns sie tödlich verwundet. Die Göttin Diana sorgt dafür, daß die Amazone nach ihrem Tode trotz ihrer Untreue in ihrer Heimat in einem stattlichen Grab Ruhe und ewigen Ruhm findet, während ihr Mörder mit dem Tode büßt und sein Leichnam im Staub des Schlachtfeldes ruhmlos zurückbleibt. (Aen. XI 595-807)

Statt einer umfassenden Betrachtung der Charakterzüge und Lebensumstände Camillas, die eigentlich notwendig wäre um ihre von Widersprüchen über-

zufällig muß z. B. Binder alle anderen Stellen in der Aeneis, wo horrendus-a vorkommt, ob auf Personen oder Sachen bezogen, negativ übersetzen (Aen. II 222. III 26. IV 558. III 658. III 679. III 712. IV181. IV 454. VI 10. VI 98. VI 285. VI 288. VI 299. VI 327. VII 78. VII 173. VII 323. VII 568. VIII 565. IX 112. IX 521. IX 633. XII 700). Dies gilt auch für Verg., Georg. II 387 und sonstige Stellen in der lateinischen Literatur, wo horrendus und ähnliche Formen häufig als Ausdruck primär positiven Gefühls gedeutet werden Petron. XXI 3. CXXIII $240 \mathrm{ff}$.. Cic., Att. VIII 9. 4. Vgl. dazu die richtigen Bemerkungen bei Horsfall, 1988, z.St. Ein Synonym für horrenda virgo ist aspera virgo (XI 664: ,grimmige Jungfrau“ Binder), was keinen Zweifel über die Bedeutung auch von dem Synonym horrenda übrig lässt. Vgl. pestis aspera $=$ die unterirdische Allecto VII 505.

${ }^{3}$ Verg., Georg. II 136 ff. bes.167-176. Aen. VIII 603-613. Plin., Nat. 3, 38-42. 12, 5, 2.

${ }^{4}$ XI 585: lacessere Teucros. 
schattete Existenz zu verstehen, beschränke ich mich im engen Rahmen eines Vortrags auf die Analyse von zwei grundlegenden Momenten, auf zwei Momente, die sich am allerersten Anfang dieses Lebens sich ergeben und als solche, wie es öfters zu beobachten ist, sich für das ganze Leben als bestimmend erweisen. Ich werde ihr Verhältnis zur Lanze sowie zum Fluß ihres Rettungsfluges, dem Amasenus, ja zu den Gewässern ihrer Umgebung im Allgemeinen näher in Augenschein nehmen.

\section{II}

Eine Lanze, die zum Schicksal wird

a

Die Lanze spielt im Leben der einige Wochen alten Camilla nur als Wurfinstrument eine Rolle, das heißt nur mit ihrem Holzteil und nur in positiver, lebensrettender Funktion. Lanzenstiel und Säugling erscheinen dabei als unlösbare Einheit, ja diese Unlösbarkeit bildet die Existenzgrundlage des Kindes. Eine Einheit, in der nicht der Mensch, sondern das Instrument dominiert, das Kind hängt von der Lanze ab, im wörtlichen Sinne des Wortes. Nicht eine virgo cum hasta fliegt somit über den todbringenden wilden Strom, sondern eine hasta cum virgine (XI 565), eine Lanze in Begleitung einer Jungfrau ${ }^{5}$.

\section{b}

Die Lanze ist auch an späteren Wendepunkten in Camillas Leben präsent. Die einzelnen Vorkommen bilden dabei in ihrem Nacheinander insgesamt ein überraschend sinnvolles Ganzes. Die nächste bemerkenswerte Szene ist das Auftreten der Jungfrau im Rahmen der Heerschau der Italiker, als letzte, als Anhang, der zugleich aber auch Höhepunkt ist. Nach Erwähnung von Purpurmantel, goldener Haarspange, besonderem Köcher folgt die Beschreibung der Lanze als Endpunkt, in einer besonderer Aufmerksamkeit würdigen Weise: pastoralem praefixa cuspide myrtum $=($ Sie hält in ihrer Hand $)$ einen vorne mit einer Eisenspitze versehenen Hirtenstab aus Myrtenholz (VII 817). Es handelt sich dabei um einen zur Lanze umfunktionierten Hirtenstab, durch Hinzufügung einer eisernen Spitze. ${ }^{6}$ Daraus ergibt sich von selbst eine dominante Position

\footnotetext{
${ }^{5}$ Dabei ist die Charakterisierung eines unreifen Kindes als Jungfrau hier eine Antizipation der Zukunft.

${ }^{6}$ Ausnahmsweise wird auch ein Hirtenstab mit einer eisernen Spitze erwähnt: Plin., Nat. 36,127. Hier ist aber eine eiserne Spitze für den Hirtenstab eine in den Textzusammenhang passende Notwendigkeit, da es um die Entdeckung des Magnetsteins durch einen Hirten mit einem
} 
des hölzernen Stabes und der bloße Zusatzcharakter der Spitze. Diese Position wird aber noch durch die Gestaltung der Wortfolge weiter hervorgehoben. Die Erwähnung der Lanze bildet nämlich den Schluß der Beschreibung des Auftritts der Jungfrau, und dadurch gleichzeitig auch der ganzen Heerschau, ja den Endpunkt des ganzen siebten Gesanges. Innerhalb der Beschreibung der Lanze aber kommt die Schlußposition dem Holzteil myrtus zu und nicht dem Instrumententeil, das die Lanze zur Waffe macht, der Lanzenspitze, cuspis. Myrtus erscheint auf diese Weise als absolutes Ausklangswort eines ganzen Gesanges, was ihm unvermeidlich herausgehobene Aufmerksamkeit sichert.

Diese Dominanz des Holzteils gegenüber der Waffenspitze weist zurück auf die anfängliche Flußszene, ist aber hier nicht mehr absolut, sondern durch die Erwähnung der Lanzenspitze eingeschränkt, deren Vorhandensein andrerseits schon auf die späteren Kämpfe voraus weist, in denen die Funktion der Lanzenspitze voll zur Geltung kommen wird. Flußszene und Heerschauszene sind aber auch durch einen weiteren Bezugspunkt besonders verbunden. Beide Male wird der Lanzenstiel als für die vorgesehene Funktion geeignet beschrieben, jedoch auf jeweils andere Weise. Bevor das Kind am Amasenus-Ufer an die Lanze gebunden wird, wird deren zum Lastentragen geeignete Solidität betont. In ähnlicher Weise betont die Hervorhebung des Myrtenholzes der Lanze ihre Eignung als Element eines feierlichen Aufmarsches, dessen Grundcharakterzüge Schönheit und Friedlichkeit sind: Denn zu dieser Schönheit trägt das Myrtenholz der Lanze in der Hand Camillas bei, mehr als deren Eisenspitze. Daß die Myrte in Rom nicht zuletzt ein Symbol für Schönheit ist, ergibt sich schon daraus, daß sie Attribut der Göttin Venus ist, deren Namen bereits Schönheit, Anmut (als Anlaß für Liebe) bedeutet. Auf der anderen Seite hat Myrtenholz auch einen Symbolwert des Friedens und verstärkt damit auch diesen „,friedlichen" Charakter der Heerschau. Denn Venus ist als Göttin der Liebe per definitionem auch Göttin des Friedens. Um einen heute modischen Spruch anzuwenden: ,she makes love, not war“. ${ }^{7}$ Weiterhin ist sie Gottheit des Friedens auch als Venus Libitina, Göttin des Todes d.h. der ewigen Ruhe. ${ }^{8}$ Die Myrte als At-

dafür geeigneten, d.h. mit einem Stück Eisen versehenen Hirtenstab geht. Tareton 1989, 267. Für bloßes Myrtusholz als Symbol für friedliche Tätigkeit, das dem Eisen als Symbol für Krieg entgegengesetzt wird, findet sich ein schönes Beispiel bei Ov., Am. I 1, 27.

${ }_{7}$ Lucr. I 28-40, bes. 31 f.: tu sola potes tranquilla pace iuvare / mortales. Germanicus, Fragmenta Prognosticorum, frg. IV 88: Sin Venus ingressa est spatiosi sidera cancri / Pacem mundus habet.

${ }^{8}$ Die Existenz einer Venus Libitina ist ein umstrittenes Problem, auf das hier nicht eingegangen werden kann. $\mathrm{Da} ß$ sie mehr war als eine zufällige Namenskombination, beweist die Parallele einer griechischen Aphrodite Epitymbia, = „Aphrodite am Grabe“. Eine solche Existenz hat auch einen inneren Sinn. Denn beide Vorstellungen, Liebe und Tod sind verschiedene Formen der 
tribut der Venus besitzt auch all diese Eigenschaften und ist damit in einem schönen friedlichen Aufmarsch an ihrem richtigen Platz.

Nun ist aber die Friedlichkeit eines militärischen Aufmarsches nur momentan und diese Einschränkung wird gut zum Ausdruck gebracht durch die zwar momentan nebensächliche, aber in der Zukunft hauptsächliche Präsenz der Eisenspitze, die darauf hinweist, daß die Friedlichkeit nur gegenwärtig, vorübergehend ist, keine Zukunft hat. Es ist für die hier behandelte Stelle von besonderem Interesse, daß die Myrte in Rom auch sonst als Symbol eines durch Krieg eingeschränkten Friedens, besser gesagt eines durch Frieden eingeschränkten Krieges vorkommt und zwar im wirklichen Leben. Sie galt als Symbol eines ohne Blut Vergießen erreichten Sieges. ${ }^{9}$ Der gelehrte Römer Gellius erklärt dies damit, daß der myrtus ein Attribut der Venus ist ${ }^{10}$. Durch diese römische Tradition der Myrte wird auch in unserem Falle die theoretische Möglichkeit offen gelassen, daß auch Camilla als Trägerin von Myrte einen sanften Krieg führen wird, was dann anders kommt. ${ }^{11}$

c

Ein drittes Mal spielt die Lanze in der Camilla-Geschichte im Kampfgeschehen selbst eine Rolle, hier ihrer eigentlichen Natur als Kampfinstrument endlich gerecht werdend, gleichmäßig in ihren beiden Teilen, zum Werfen auf den Feind mittels ihres Holzteils und zum Verwunden und Töten mit ihrer Eisenspitze. ${ }^{12}$ Der Holzteil erhält kein Übergewicht mehr, sei es voll, sei es halbwegs, als Flugmittel oder als Mahnung an Schönheit und Friedensmöglichkeit.

Befriedigung und des Friedens. Der Zweck einer Liebesvereinigung ist die Erreichung körperlichen und seelischen Friedens wie als Wesen des Todes die ewige Ruhe gilt: „requiescat in pace!“ Enn., Trag. 364. Cic., Tusc. I 44,107. Ov., Am. III 967. Metam. IV 166. *Sen., Epigr. c .415 (Anth. Lat. I 1), 5. Petron., Sat. LXXI 12. Mart., Epigr. I 93,1. VI 18,1. X 61,1. Aus solcher Überlegungen heraus teile ich nicht die herrschende Skepsis hinsichtlich einer echten römischen Venus Libitina.

Die Myrte als Todessymbol unabhängig von Venus: Verg., Aen. III 23 ff. Vgl. auch 41: ,parce sepulto"!!

${ }^{9}$ Plin., Nat. XV 125: P. Postumius Tubertus ... primus omnium ovans ingressus urbem est, quoniam rem leniter sine cruore gesserat, myrto Veneris victricis coronatus incessit optabilemque arborem etiam hostibus fecit. Er war consul 503 v.Chr: Liv. II 16.

${ }^{10}$ Noctes V 6,20-21: Ovandi ac non triumphandi causa est, cum aut bella non rite indicta neque cum iusto hoste gesta sunt aut hostium nomen humile et non idoneum est, ut servorum piratarumque, aut deditione repente facta inpulverea, ut dici solet, incruentaque victoria obvenit. Cui facilitati aptam esse Veneris frondem crediderunt, quod non Martis, sed quasi Venerius quidam triumphus foret.

${ }^{11}$ Torrão $1993,121 \mathrm{f}$

${ }^{12}$ XI 650, 664, 666, 674, 676, 685, 691. 
Im Gegenteil: die Eisenspitze ist von überwiegender Wichtigkeit als letztendliche Verwirklicherin des eigentlichen Zweckes der ganzen Waffe, der Verwundung und Tötung. So vollendet sich die verhängnisvolle funktionale Entwicklungsreihe der Lanze hier vom Mittel der Lebensrettung über Symbol blühenden, obwohl potentiell bedrohten Lebens ausschließlich zum Instrument der Lebensvernichtung. Sie ist die Hauptwaffe Camillas im Kampf, und jede ihrer geworfenen Lanzen tötet. ${ }^{13} \mathrm{Ja}$ eine Lanze wird zur Vollenderin ihres tödlichen Schicksals selbst, ein Ereignis besonderer Aufmerksamkeit wert.

hasta sub exsertam ... perlata papillam / haesit virgineumque alte bibit acta cruorem $=$ der Speer unter die entblößte Brust traf, dort steckenblieb und tief eingedrungen, das Blut der Jungfrau trank (Aen. XI 803-4). An dieser Wunde stirbt Camilla, die aber keine gewöhnliche, sondern eine besondere Wunde ist. Dieses Besondere wird durch das Attribut „acerbus“, „bitter“ ausgedrückt: nunc vulnus acerbum conficit $=$ jetzt verzehrt mich die bittere Wunde. Bitter ist sie deswegen, weil sie Camilla entstellt, sie zur tristi mulcatam morte Camillam macht, der „,vom düsteren Tod entstellten Camilla“ (XI 839).Wenn sie aus dem Leben cum gemitu ... indignata sub umbras $=$ mit einem Seufzer entrüstet hinab zu den Schatten scheidet (XI 831), so mag in ihrer Entrüstung auch diese Besonderheit ihrer Wunde eine Rolle gespielt haben. ${ }^{14}$ Diese Wendung ist dem Ende des Patroklos und des Hektor in der Ilias nachgebildet ${ }^{15}$ und wird auch auf das Ende des Turnus angewandt. ${ }^{16}$ Vergil hat die Wendung intensiviert: in der Ilias ist von keiner besonderen (,bitteren“) Wunde die Rede und dementsprechend auch von keiner Entrüstung. Die griechischen Helden scheiden nur klagend und der Grund für die Klage ist, daß sie ihr Heldenleben zu früh verlassen müssen. ${ }^{17}$ Dies mag auch Camilla so empfunden haben. Der Grund der nur bei ihr vorhandenen Entrüstung ist aber das, was über Verlust von jungem Leben und Heldentum hinausging, die Entstellung ihrer Erscheinung. Worin besteht aber dieser unwürdige Zustand ihres sterbenden Körpers? Er ist nicht nur in ästhetischem, sondern auch in höherem Sinne entstellt, entheiligt, in seiner sakralen Unberührbarkeit verletzt. Nach den Worten der Göttin Diana

\footnotetext{
${ }^{13}$ XI 676 f.: quotque emissa manu contorsit spicula virgo / tot Phrygii cecidere viri.

${ }^{14} \mathrm{Zu}$ der Kombination vulnus acerbum vgl. Ov., Pont. I 3,8. Metam. V 63. Stat., Theb. IX 320. Claudian., Carm. mai. III 182.

${ }^{15}$ Il. XVI 857 f. XXII $362 \mathrm{f}$.

${ }^{16}$ XII 952. Alle diese Parallellitäten und Zusammenhänge haben ihren tieferen Sinn, auf den hier nicht näher eingegangen werden kann. Sicher ist soviel, daß die Parallelität in der Ilias das Verhältnis von ursprünglichem Täter und Rächer zum Ausdruck bringt, während es in der Aeneis ein Ausdruck von Zusammengehörigkeit in irgendwelchem tieferen Sinne ist.

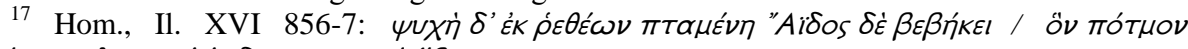

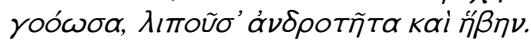


hat ihr Mörder sacrum violarit vulnere corpus, einen geweihten Leib durch Wunden misshandelt (591). Viele Momente weisen darauf hin, daß diese „bittere Entstellung eines unberührbaren Körpers durch eine Wunde" als symbolische Defloration zu verstehen ist. Die nackte Jungfrauenbrust als Zielpunkt verleiht dem Geschehen von vornherein eine erotische Nuance, sie eignet sich sehr gut als durch verbale Diskretion verursachte stellvertretende Vergegenwärtigung eines den natürlichen Zielpunkt einer Defloration bildenden weiblichen Körperteils. ${ }^{18}$ Denn durch gewaltsames Eindringen verursachte Blutung ist auch das Kennzeichen wirklicher Defloration. Die Lanze als phallisches Symbol ist eine verbreitete Erscheinung, ${ }^{19}$ wie eine Wunde für das weibliche Schamteil. ${ }^{20}$ Camilla begibt sich auf das Schlachtfeld, setzt sich einer möglichen Verwundung in dem Alter aus, in dem römische Jungfrauen heirateten d.h. ihre Unberührtheit einbüßten, ja sogar quasi an Stelle eines solchen Geschehens. ${ }^{21}$ All dies spricht für eine solche symbolische Bedeutung von Camillas Verwundung. Der Dichter Ausonius verstand Vergil congenialiter, als er in seinem cento nuptialis „Hochzeitsflickwerk“ betitelten Gedicht, das er auf virtuose Weise ausschließlich aus Vergilzitaten zusammengeflickt hat, die Verwundung Camilla durch die Lanze zur Darstellung der Defloration in der Hochzeitsnacht verwendet hat, auf nahe liegende Weise. ${ }^{22}$ Im Hintergrund mag eine gewisse Rolle gespielt haben die bedeutende Rolle der weiblichen Brust im Erscheinungsbild sowie in der volksetymologischen Deutung des Namens der griechischen Amazonen. ${ }^{23}$

Nicht zuletzt auf Grund dieser symbolischen Rolle der Lanze darf festgestellt werden, daß der Lanze in Camillas Lebensgeschichte und in deren Deutung eine grundlegende Rolle zukommt, wenn die diesbezüglichen Hinweise manchmal auch nur in für Vergil charakteristischen Weise zwischen den Zeilen verborgen sind, ein Element der so genannten „Zweiten Tiefe“ seines Werkes bilden. $^{24}$

Dies ist keineswegs überraschend, wenn man die außerordentliche Bedeutung der Lanze im Leben der Römer in Betracht zieht. Dieses Objekt galt nicht

\footnotetext{
${ }^{18}$ Fratantuono - Osker 2010, 112.

${ }^{19}$ Köves-Zulauf 1988 (1978) 268. Idem 2013, 228.

${ }^{20}$ Ll. cc.

${ }^{21}$ L. c.

${ }^{22}$ Carmen $9,118$.

${ }^{23}$ Häufig wird das Wort Amazone etymologisch aus dem griechischen Wort für Brust, $\mu \propto \zeta o ́ s$, abgeleitet, s. Köves-Zulauf 1988 (1978) 300, 432 Anm. 82.

${ }^{24}$ Köves-Zulauf 1988 (1978) 269, 198, Anm. 55. Anders Horsfall 1988, 44 f.: „Il fatto che un poeta abbia letto molto, anche moltissimo, non implica necessariamente anche un gusto diffuso per l'arcano o l'esoterico“".
} 
nur als „die Summe der Bewaffnung“ sondern auch die „der Herrschaft“ summa armorum et imperii, ${ }^{25}$ ersteres gerade wegen ihrer Zweiteiligkeit von Schaft und Spitze, was sie sowohl für Fern- als auch für Nahkampf geeignet, sozusagen zur absoluten Waffe, machte. Darin wurzelte auch ihre Rolle als Herrschaftsinstrument, dem bei anderen Völkern in dieser Rolle üblichen Szepter vergleichbar. Was aber insbesondere Vergilius betrifft, ihre quasi Personifikation in der Szene der symbolischen Defloration Camillas ist nicht ohne weitere Parallelen. Turnus spricht zu seiner eigenen Lanze als zu einer Person, betet zu ihr und erklärt sie zur Garant des Sieges. Der Etrusker Mezentius aber erklärt, daß die Lanze in seiner Hand für ihn die Gottheit ist. ${ }^{26}$ Letzten Endes verbirgt sich hinter all diesen Personifikationen auch der Gott Mars, der für die Römer bekanntlich sich in einer Lanze verkörperte. ${ }^{27}$

III

Camillas Umwelt

Ein Fluß, der Amasenus spielt im Leben Camillas eine grundlegende Rolle. Die Überquerung dieses Flusses als Neugeborene begründet ihre Existenz, ihre Kindheit und erste Jugendzeit verbringt sie in einer von Menschen verlassenen Wildnis jenseits dieses Flusses. Daß die auf diese Weise grenzbildende Funktion eines Flusses kein Zufall ist, zeigt der Umstand, daß schon die griechischen Amazonen, deren Vorbild nicht ohne Einfluß auf Vergil war, jenseits eines Flusses verortet wurden. ${ }^{28}$ Hinzukommt, daß für Völker, die auf der anfänglichen Stufe ihrer Entwicklung leben, das Jenseits häufig im ursprünglichen Sinne des Wortes jenseits eines Flusses, des Flusses sich befindet. Es ist kein $\mathrm{Zu}$ fall, daß noch nach antiker Weltsicht verschiedene Flüsse und Gewässer, Styx, Acheron, Kokytos, die Grenze zwischen Diesseits und jenseitiger Unterwelt bilden. Eine weitere wesentliche Verbindung des Amasenus mit der italischen Amazone Camilla ergibt sich aus der Beachtung des Flussnamens. Es ist schwer, nicht zu bemerken, daß der Name Amasenus akustisch an das Wort Amazon anklingt. -enus- wird dabei mit Recht als Adjektiv bildendes Ableitungssuffix angesehen, woraus sich als Grundwort ein Amasus ergibt ${ }^{29}$ und für Amasenus der Sinn „zu Amasus gehörig“. Was auch immer Amasus bedeuten

\footnotetext{
${ }^{25}$ Festus 55,9-10 (L.) 63 (M.); Köves-Zulauf 2013, 219-241, bes. 221-226.

26 Köves-Zulauf o. c. $224 \mathrm{f}$.

${ }^{27}$ Latte 1960, 114.

${ }^{28}$ Der Kleine Pauly s.v. Amazones 1, 292,3. Strabon I 3, 7 (C 52) (Am Thermodon, in einer Sumpfgegend). XI 5,1 und 4 (C. 502-3) (am Mermadalis).

${ }^{29}$ Wikipedia s.v. Amaseno, 7 von 18: „Il nome Amaseno deriva da un piccolo fiume denominato 'Amasenus' il quale si ipotizza che potrebbe derivare a sua volta da un nome di città Amasos".
} 
mag, es liegt auch silbenmäßig noch näher an Amazon und ordnet sich noch mehr ein in die Gewohnheit Vergils, die Zusammengehörigkeit von Phänomenen durch nur in Kleinigkeiten voneinander abweichenden Namen zu signalisieren, ${ }^{30}$ eine der Möglichkeiten zur Herstellung der berühmten zweiten Sinnebene vergilischer Dichtung. Die Bedeutung des Flusses Amasenus im Leben Camillas wird aber weiter ersichtlich, wenn der Text der Geschichte mit der Landkarte des alten Latium zusammengehalten wird. Aus einer solchen Projektion ergibt sich zwangsläufig die Erkenntnis, daß Vater und Tochter aus Privernum fliehend den Amasenus in östlicher Richtung überqueren mussten. Des weiteren aber, daß Camilla von jenseits dieses Flusses auf das Feld der Schlachten in der Nähe der Stadt Laurentum ziehend erneut über diesen Fluß ihren Weg nehmen musste, diesmal in westlicher Richtung, ob direkt, oder auf dem Umweg über Privernum. Wie das erste Hindurchgelangen über den Amasenus die dianische Existenz Camillas begründete, so wurde auf diese Weise das zweite Überqueren der „Rubicon“ der Beendigung dieser Existenz. Nicht ohne Bedeutung bleibt auch der Umstand, daß sie auf diesem Wege zur Parade und Schlacht zwangsläufig als aus Richtung der pomptinischen Sümpfe kommend erscheinen musste.

Die italische Amazone zeigt aber auch darüber hinaus weitere Affinitäten mit der Welt der Gewässer. Ein solcher Hinweis ist der Name ihres Vaters, Metabus, eine illyrische oder griechische Bildung, mit der Bedeutung ,der zwischen den Wässern". ${ }^{31}$ Ein solcher Name kommt dem Herrscher von Privernum mit vollem Recht zu, angesichts der Tatsache, daß diese Stadt am Zusammentreffen vom Amasenus und einem Nebenfluß lag. Keine einmalige Situation in dieser Gegend wie solche Städtenamen wie Interamna, Interamnium es zeigen, mit der Bedeutung „Zwischen Flüssen liegend“. Denselben Namen Metabus trägt auch der griechische Gott Poseidon, ${ }^{32}$, dessen Gestalt in Italien schon seit geraumer Zeit mit der des autochthonen Neptunus verschmolzen war, ${ }^{33}$ der ursprünglich in den auf der unbeweglichen Erde sich fortbewegenden Wasser-

\footnotetext{
${ }^{30}$ S. den Namen Camilla als Hinweis auf den Namen der Mutter Casmilla: matrisque vocavit / nomine Casmillae mutata parte Camillam: „nannte sie nach dem Namen ihrer Mutter Casmilla leicht verändert Camilla“ (Binder). Ähnlich verweist der Name der braven Gefährtin Tulla (XI 656) auf den Namen der bösen Tullia Tochter des Servius Tullius (Der Kleine Pauly 5997,57 ff. s.v. Tullius) und Larina virgo (XI 655) auf (Acca) Larentia „nobilissimum id temporis scortum“, die seinerzeit berühmteste Hure (Macrobius, sat. I 10, 13), all dies zugleich Beispiele für die Methode Vergils, Traditionen kontrapunktisch zu verwenden.

${ }^{31}$ Kretschmer, 1923, 92 ff. Altheim 1931, 24 ff. Anders Horsfall 1988, 39: „Metabo come padre di Camilla non era necessiaramente un elemento essenziale per nessuna delle due figure“.

${ }^{32}$ Altheim 1931, 21 ff.. Idem $1956^{2,}$ 1,12. 2,17.

${ }^{33}$ Latte 1960, 131; Dumézil 1966, 381.
} 
massen als deren Herr existiert ${ }^{34}$. Auf diese Weise erscheint Camillas Vater als menschliches Abbild dieses Wassergottes, ja nach einigen Forschern sogar als dessen Verkörperung ${ }^{35}$.

Camilla ist aber nicht nur das Kind eines mit dem Wassergott gleichnamigen Vaters, sondern hat neben sich sogar einen vorrangigen Kampfgefährten, der expressis verbis und dies viermal ${ }^{36}$ als leiblicher Sprössling des Neptunus, Neptunia proles, charakterisiert wird. In dieser Eigenschaft trägt er denselben Namen wie Camillas Vater mit zwei kleinen Änderungen, Messapus statt Metabus, was an der Bedeutung nichts ändert, gleichzeitig aber ein erneutes Beispiel der erwähnten vergilischen Methode darstellt, die Zugehörigkeit von zwei Phänomenen tacendo loquens anzudeuten.

Was mag Grund und Sinn eines so engen Verhältnisses Camillas mit der Welt der sie umgebenden Gewässer sein? Bevor eine Antwort auf diese Frage versucht wird, empfiehlt es sich, die Gestalt der zweiten selbstständig agierenden italischen kriegerischen Jungfrau in der Aeneis in Augenschein zu nehmen, die die Schwester des Feldherrn Turnus ist und Iuturna genannt wird. Besitzt sie ähnliche Eigenschaften wie Camilla, zeigt auch sie namentlich Verbundenheit mit Gewässern ihrer Umgebung?

$\mathrm{B}$

Iuturna virago

Schon ein flüchtiger erster Blick lässt erahnen, daß die zwei nach männlicher Art kriegerisch tätigen jungen Frauen nicht zufällig in ähnlicher Weise tätig sind, sondern daß dies in ihrem Charakter und in ihren Lebensbezügen seine Grundlage hat. Die zwei Appositionen, virgo und virago ${ }^{37}$, die ihr Wesen zum Ausdruck bringen sollen, sind lautlich ähnlich, wortbildungsmäßig von vergleichbarer Struktur und beide klingen an das Wort vir an, entsprechend der männlichen Rolle der beiden jungen Frauen.$^{38}$ Virago ist nichts anderes als eine inhaltliche Variante von horrenda virgo in ein einziges Wort gefasst. Nicht zufällig spricht des Weiteren ihre Bezugsperson Turnus sie beide mit dem gleichen Kompliment decus an, Camilla als decus Italiae, Iuturna als decus

\footnotetext{
${ }^{34}$ Der Kleine Pauly 4,1076 s.v. Poseidon.

${ }^{35}$ Altheim, $1956^{2}, 17$.

${ }^{36}$ Aen. VII 691. IX 523. X 352. XII 127.

${ }^{37}$ Camilla virgo: VII 806, XI 507, 508, 536, 664. Iuturna virago: XII 468.

${ }^{38}$ Die richtige Etymologie der beiden Wörter ist ein bis jetzt ungelöstes Problem. Das Nebeneinander von virgo und virago hat seine Parallelen in der Paarung von Camilla/Casmilla und Tulla/Tullia wobei die zweite Form nicht nur vokalmäßig, sondern auch inhaltlich eine Steigerung ist: Casmilla ist weiblicher als Camilla, Tullia mörderischer als Tulla, virago mannbezogener als virgo.
} 
fluviorum (XII 142). Dies zeigt schon, daß beide zwar vergleichbar, aber nicht identisch sind; andrerseits, daß Iuturnas Gestalt tatsächlich weitere Aufklärung auch darüber erwarten lässt, was das Verhältnis Camillas und ihr ähnlicher Gestalten zu Gewässern auf sich hat.

Iuturnas Geschichte lässt sich folgendermaßen kurz zusammenfassen:

Latiner und Trojaner bereiten einen Vertrag vor, auf dessen Grundlage Turnus und Aeneas statt eines allgemeinen Schlachtgetümmels einen Zweikampf ausfechten würden. Die Göttin Iuno beabsichtigt, diesen Plan zu vereiteln, da sie davon ausgeht, daß der Zweikampf mit dem Tod ihres Schützlings, des Turnus enden würde. Sie beauftragt daher Iuturna, durch Verursachung eines allgemeinen Durcheinanders den Zweikampf zu verhindern. Der Plan gelingt. Im beginnenden allgemeinen Kampf nimmt Iuturna die Gestalt des Metiscus, Wagenlenkers des Turnus an und verhindert durch Hin - und Herlenken des Wagens, daß Turnus und Aeneas aufeinander treffen. Am Ende bringt Iupiter, im Einverständnis mit Iuno, durch Aussenden eines unterirdischen Vogels gegen Turnus, dem vorbestimmten Fatum entsprechend, Iuturna zur Kenntnis, daß sie ihren dem Tode geweihten Bruder verlassen muß. Die virago Iuturna verlässt mit bitteren Worten das Schlachtfeld, in einer berühmten Szene der Selbstbezichtigung, ${ }^{39}$ um in einem tiefen Fluß unterzutauchen. ${ }^{40}$

Ein erster gemeinsamer Zug der beiden Erzählungen von nach Männerart kämpfenden jungen Frauen ist die Anwesenheit einer die weibliche Existenz repräsentierenden und beschützenden Gottheit in ihrer Geschichte, Diana in der einen, Iuno in dem anderen Fall. Beide sind Göttinnen weiblichen Seins, beide Geburtsgöttinnen, jedoch unter zwei verschiedenen Aspekten. Diana repräsentiert und beschützt die weibliche Existenz grundlegend in deren natürlichen Erscheinungsformen, während Iuno vor allem für das frauliche Sein als soziale Erscheinung zuständig ist. Daß dieses Nebeneinander der zwei Göttinnen keine oberflächliche Zusammenstellung ist, sondern eine erst zusammen das Ganze des weiblichen Wesens reflektierende notwendige Zweiheit, lassen räumliche und historische Tatsächlichkeiten erkennen. Iuno und Diana besaßen in Rom Tempel auch in unmittelbarem räumlichem Nebeneinander im circus Flaminius, gleichzeitig gelobt und zusammen geweiht $179 \mathrm{v}$. Chr. durch denselben M. Aemilius Lepidus. ${ }^{41}$

Der Eigenart der zwei Göttinnen und ihres Verhältnisses zueinander entspricht der Charakter ihrer Schützlinge und deren Nebeneinander. Diana hat es mit einem Mädchen zu tun, das in der Wildnis der Natur aufgewachsen ist, diese Umwelt nur gegen den Willen ihrer Göttin verlassen kann, während Iunos

\footnotetext{
${ }^{39}$ Perkell 1997, 257-286.

${ }^{40}$ Aen. XII 10. 134-886.

${ }^{41}$ Liv. XXXIX 2.8. XXXIX 2,11. XLII-LII, 1-2. Latte 1960, 417.
} 
Schutz einer jungen Frau gilt, deren weibliches Schicksal durch ihre gesellschaftliche Stellung bestimmt ist und ein gesellschaftliches Ereignis, einen Krieg, zu beeinflussen ihre Aufgabe darstellt. Die körperliche Unversehrtheit Camillas ist ein naturgemäßes Phänomen, sie ist virgo im biologischen Sinne des Wortes, während Iuturna biologisch keine Jungfrau mehr ist, als ehemaliges Opfer von Jupiters Gewalt, gilt aber sozial als Jungfrau, da sie als junge Frau Mann ablehnend lebt, ja sogar als Mann. Dies beinhaltet ihr Kennwort virago. Des Weiteren ist Camilla nur eine gottähnliche dia genannte Gestalt - als einzige in der ganzen Aeneis. ${ }^{42}$ - Iuturna demgegenüber ist Göttin im vollen Sinne des Wortes, wenn auch als ehemaliger Mensch, als Wiedergutmachungsgeschenk Iupiters. Des Weiteren sind beide Kämpferinnen durch wesentliche Verbundenheit mit dem italischen Feldherrn Turnus charakterisiert, aber auf jeweils verschiedene Art. Camilla und Turnus sind durch die Solidarität sterblicher Menschen verbunden: auch dies gewinnt Ausdruck in der Identität ihrer Todesverse. ${ }^{43}$ Iuturnas Verhältnis zu Turnus ist enger und höherwertig: Als Mensch ist sie seine Schwester, als Göttin gewährt sie ihm göttlichen Schutz. Camilla versucht am Anfang ihrer Kriegstätigkeit einen strategischen Plan Turnus nahe zu legen, ohne Erfolg. Auf jeden Fall kämpft aber Camilla an der Spitze der Reiterei, während Turnus die Leitung der Infanterie bleibt. Iuturna tritt demgegenüber in enger Einheit mit Turnus auf im selben Kampfwagen und sie bestimmt, wo der Wagen hingeführt werden soll. Die virgo kämpft auf dem Pferd, die virago in einem von Pferden gezogenen Wagen. Dies entspricht vollends der Natur ihrer jeweiligen göttlichen Patronin. Im Kultus Dianas spielt das Pferd eine Rolle, als Symboltier des Krieges ${ }^{44}$, wenn auch in negativer Form, entsprechend dem negativen, verbietenden Verhältnis der Göttin zur kriegerischen Tätigkeit. Denn im Heiligtum der Diana im nahe liegenden Heiligtum in Aricia war die Anwesenheit von Pferden verboten. ${ }^{45}$ Demgegenüber kämpft Iuturna in einem Kampfwagen und benutzt diesen in Einverständnis mit ihrer Göttin. Und der Kampfwagen ist ein repräsentatives kultisches Attribut Iunos. ${ }^{46}$ Auf Grund des bisher Dargelegten kann festgestellt werden, daß viele Charakterzüge Iuturnas Elemente wiederholen, die auch für Camilla charakteristisch waren, und sie tun dies häufig in gesteigerter oder gar kontrapunktischer Form. Eine weitere vergleichende Analyse von Wesen und Tun beider Gestalten könnte noch mehr einschlägige Erkenntnisse zu Tage fördern. ${ }^{47}$ Dafür gibt es

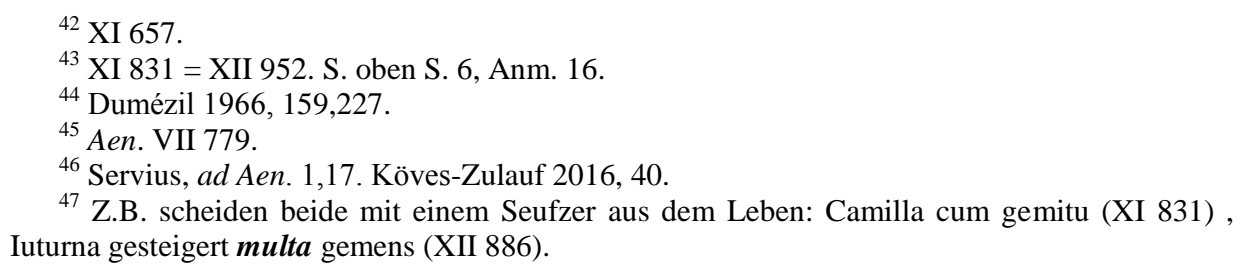


jedoch im gegebenen Rahmen keine Möglichkeit. Ein bestimmtes Motiv noch festzuhalten empfiehlt sich jedoch wegen dessen besonderer Bedeutung und dies ist das Verhältnis der beiden jungen Frauen zu dem Element Wasser, auf zwei Ebenen, einerseits als Übereinstimmung im Charakter, andrerseits als lokale Verbundenheit. Auch dies erscheint ausgeprägter bei Iuturna als bei Camilla. Camilla ist nur ähnlicher Wesensart wie Wasser, mit ihrer sagenhaften Schnelligkeit, ${ }^{48}$ Iuturna dagegen ist Wasser. Die Verbindung Camillas mit Flüssen und Gewässern (Amasenus, Pomptinae Paludes) besteht in räumlicher Nähe, Iuturnas demgegenüber in räumlicher und persönlicher Identität: sie ist die göttliche Personifikation einer heiligen Quelle in Rom und (oder) in Latium

\footnotetext{
${ }^{48}$ Camillas außerordentliche Mobilität wird in der Heerschau in fünf Zeilen geschildert, ausführlicher als jede andere ihrer Eigenschaften:

.....Camilla

agmen agens equitum..

.....adsueta....

....cursuque pedum praevertere ventos.

Illa vel intactae segetis per summa volaret

Gramina nec teneras cursu laesisset aristas,

Vel mare per medium fluctu suspensa tumenti

Ferret iter celeris nec tingueret aequore plantas.

....(Camilla, die eine Reiterschwadron führte)...

Geübt...

Im Laufe den Winden zuvorzukommen:

Sie könnte wohl über die Spitzen der Halme schnittreifen Korns fliegen

Ohne die zarten Ähren im Lauf zu beschädigen,

oder mitten durchs Meer auf brausender Flut schwebend

ihren Weg gehen, ohne ihre schnell tragenden Sohlen mit Wasser zu benetzen (Binder).

Die schnelle Bewegung wird nicht nur durch Inanspruchnahme eines Hilfsmittels, des Pferdes erreicht, sondern ist auch eine natürliche Fähigkeit der Jungfrau selbst. Diese Begabung wird unmittelbar der traditionellen auf den Raum des Hauses begrenzten Lebensweise der Frauen gegenübergestellt. Die Fähigkeit der Bewegung wird außerdem in ihrer intensivsten Form Camilla zugeschrieben, dadurch daß sie mit dem Lebewesen verglichen wird, welches zur schnellsten Art von Bewegung fähig ist, einem Vogel (volaret Z. 808-11, 721: sacer ales).

Aber auch die Bewegung auf dem Pferderücken, die Verbindung mit diesem Tier schneller Bewegung gehört zu ihren eminenten Wesenszügen. Als Säugling schon wird sie mit Stutenmilch am Leben gehalten. In der Heerschau wird ihr Berittensein vor allem anderen hervorgehoben. Sie ist in jeder Hinsicht die velox Camilla, die „schnelle Camilla“ wie der Dichter sie mit Recht nennt (760). Daß sie in Schnelligkeit ihre Feinde übertrifft, ist ein Geheimnis ihrer Sieghaftigkeit. $\mathrm{Da} ß$ nun die schnelle Bewegung ein mit dem Quell- und Flußwasser gemeinsame Eigenschaft ist, liegt auf der Hand. Eine Gemeinsamkeit, die schön durch das Pferd symbolisiert wird. Dieses Tier der Schnelligkeit ist nicht nur das repräsentative Tier Camillas, sondern das Symboltier des Gottes schnell dahineilenden Wassers, Neptunus-Poseidon.
} 
und des diese umgebenden Sees und Sumpfes. Sie versinkt nach Vollendung ihrer Aufgabe in dem hochheiligen Fluß Numicus (Aen. XII 886). ${ }^{49}$

Der Vergleich mit Iuturna bekräftigt die auf den ersten Blick vielleicht unglaublich, auf den zweiten, mag sein, grotesk erscheinende wesentliche Verbindung Camillas mit dem Element Wasser. Eine weitere begrüßenswerte Bestätigung wird durch einen weiteren Vergleich ermöglicht, mit einer Mannfrau, virago, die zwar in der Aeneis nicht auftritt, aber als Vorbild für Iuturna im Hintergrund, auf latente Weise, wahrscheinlich vorhanden ist und ihre lokale Verbundenheit mit einem Gewässer sogar durch ihren Namen offen kundtut. Es handelt sich um eine Gestalt aus den Annalen des Ennius, aus welchem Werk Vergil bekanntlich manche Anregung gewann.

\section{$\mathrm{C}$}

Paluda virago und Verwandtes

Der Polyhistor Varro zitiert im etymologischen Teil seines Werkes über die lateinische Sprache, ${ }^{50}$ nach der Deutung solcher Wörter wie camilla, subulo, Fauni, vates aus dem Werk des Ennius folgende Zeilen ${ }^{51}$ :

Corpore Tartarino prognata Paluda virago

Cui par imber et ignis, spiritus et gravis terra ${ }^{52}$

„Mit tartarinischem Körper in die Welt geborene Mannfrau Paluda,

der Regen und Feuer, Lufthauch und schwere Erde gleich sind“

Tartarinus ist eine adjektivische Ableitung aus dem Wort Tartarus und dieses hält Varro für den Namen eines unterirdischen Flusses, die virago also für die Ausgeburt eines Flusses der Unterwelt. Sie besitzt dementsprechend auch einen Körper aus Materie mit Wassereigenschaften. Denn dieses Element ist es, dem weder Regen noch Feuer, noch wehende Luft, noch Erdenschwere etwas anhaben können. ${ }^{53}$ Hier haben wir also wieder die lokale Verbundenheit einer Mannfrau mit Wasser, gepaart mit ihrer Wasserartigkeit. Dem entspricht der Name dieses Wesens. Er kann nach allen Regeln der Sprachgeschichte aus kaum einem anderen Wort als dem Wort palus „Sumpf“ abgeleitet werden und bedeutet damit nichts anderes als „Sumpffrau“. Dies mag auf den ersten Blick als sonderbar erscheinen, verliert aber viel von seiner Sonderbarkeit, wenn man

\footnotetext{
${ }^{49}$ Serv., ad Aen. XII 139. Della Corte 1972, 207 f.

${ }^{50}$ KP s.v.Varro 1134, $24 \mathrm{ff}$.

${ }^{51}$ Var., L. VII 3,37.

${ }^{52}$ Annalium incerti versus 520-521.

${ }^{53}$ Vgl. über den Neptun Sohn Messapus 692: quem neque fas igni cuiquam nec sternere
} ferro. 
bedenkt, daß mit „Sumpf“ palus in Zusammenhang gebrachte weibliche Wesen mehr als einmal in der römischen Tradition auch sonst vorkommen. Es werden erwähnt Caprae palus,${ }^{54}$ Saturae palus,${ }^{55}$ „,Sumpf der Ziege“, ,Sumpf der Sättigung" ${ }^{56}$ ein Symbol der Fruchtbarkeit neben einem Begriff der Fruchtbarkeit, ${ }^{57}$ rätselhafte, wenig erforschte Gestalten, was die Richtigkeit einer bereits vor mehr als 50 Jahren getroffenen Feststellung von G. Dumézil bestätigt: „Die Dämonologie der Römer ist wenig bekannt“.58 Sehr beachtenswert ist dabei, daß beide erwähnten mit Sümpfen verbundenen Gestalten in kriegerischem Zusammenhang vorkommen. Neben Caprae palus hält Romulus eine Heerschau ab, die mit seinem Tod endet, ${ }^{59}$ und aus der Gegend von Saturae palus ziehen Männer in den Krieg in Vergils Aeneis (VII 801). Dieser Sumpf weist auch einen Berührungspunkt mit der im Heerschau auftretenden Camilla auf, der schwer zu deuten ist und erneut die Schwierigkeit dieses dämonologischen Themas zeigt. Sie tritt nämlich unmittelbar im Anschlu $\beta^{60}$ an die Ankömmlinge aus der Gegend auf „wo Saturas düsterer Sumpf sich erstreckt, wo der eiskalte Ufens durch tiefste Niederungen seinen Weg bahnt und sich ins Meer ergießt $\mathrm{t}^{\text {“ }}{ }^{61}$ Ist dieses Nacheinander purer Zufall oder reflektiert es den Umstand, daß Camilla, wie oben festgestellt, aus der Gegend der pomptinischen Sümpfe gekommen sein muß, als deren Teil die Saturae palus erscheint? Interessant ist

\footnotetext{
${ }^{54}$ Liv. I 16,1. Ov., Fast. II 491. Flor., Epit. I 16,1. Aur. Vict., De vir. ill. 2,13.

${ }^{55}$ Verg., Aen. VII 801. Sil. VIII 379.

${ }^{56} \mathrm{Zu}$ dieser Bedeutung des Wortes satura s. z.B. Verg., Ecl. X 77. Ov., Fast. V 498. Met. XV 498. Colum., Res rust. VI 23.

${ }^{57} \mathrm{Ob}$ es sich um dieselbe Gestalt unter zwei verschiedenen Namen handelt, Satura = Capra? Tatsache ist, daß satura häufig als Attribut von capra vorkommt wozu s. vorige Anmerkung.

${ }^{58}$ La religion Romaine archaïque. Paris,1966, 231.

${ }^{59}$ Liv. I 16,1. Ov., Fast. II 491 ff. Flor. I 1,16. Aur. Vict, De vir. ill. II 13.

${ }^{60}$ Hos super advenit Volsca de gente Camilla $=$ „Zu diesen gesellte sich endlich, vom Stamm der Volsker, Camilla" (VII 803).

${ }^{61}$ qua Saturae iacet atra palus gelidusque per imas / quaerit iter vallis atque in mare conditur Ufens.

Silius Italicus beschreibt diese Gegend folgendermaßen Pun. VIII $379 \mathrm{ff}$.:

Qua Saturae nebulosa palus restagnat et atro

liventes caeno per squalida turbidus arva

cogit aquas Ufens atque inficit aequora limo

„wo Satura's nebliger Sumpf übergießt und

seine von Schmutz bleifarbigen Wasser der stürmische Ufens

durch die von Dreck starrenden Felder zwingt

und das Meerwasser mit Schlamm entstellt"“.
} 
auch die Zweiheit Saturae palus - Ufens. Denn auch Letzterer trägt die Vorstellung der Fruchtbarkeit in seinem Namen. ${ }^{62}$

Die bisherige Ansammlung von (Jung)frauen im Zusammenhang mit Wassergegenden und Krieg, - Camilla, Iuturna, Paluda, Satura, Capra - ist Anlaß genug, um nach weiteren eventuellen Parallelgestalten Ausschau zu halten. Wobei ich mich hier auf die bloße Erwähnung von weiteren Namen als mögliche Untersuchungsgegenstände beschränken muß, die Möglichkeit eines negativen Ergebnisses einer eventuellen genauen Untersuchung offen lassend.

Hier ist zunächst die Göttin Feronia, deren Hain in der Nähe der Saturae palus auch als Ausgangspunkt der vor Camilla ankommenden Kriegerschar erwähnt wird (VII 800). Eine vielseitige und auch an vielen anderen Orten in Mittelitalien verehrte Gottheit, die jedoch auch Quellgöttin ist und gelegentlich Iuno Virgo genannt wird. ${ }^{63}$ Oder die Personifikation der stadtrömischen Wasserleitung unter dem Namen Aqua Virgo, die gerade am Tempel Iuturnas am Marsfeld endete und die der spätantike Verfasser eines Epigramma Bobiense, vielleicht der Dichter Naucellius paludigenis perlucida Virgo fluentis $=$,aus sumpfgeborenen Flüssen (entstandene) durchsichtig klare Jungfrau“ nannte. ${ }^{64}$ Oder schließlich die sehr alte (7. Jh. v.Chr.) Göttin Marica, nach Vergil Mutter des Urkönigs Latinus (Aen. VII 47), deren Heiligtum mitten in einem Sumpf lag, der palus Maricae genannt wurde (!), ${ }^{65}$ und deren Namen tadellos aus dem Wort mare „Meer“ abgeleitet werden kann. ${ }^{66}$

Die auf solche Weise aufscheinende große Bedeutung von fließenden sowie stehenden Gewässern, nicht zuletzt von Sümpfen, mag als sonderbar erscheinen. Der Hinweis auf zwei wichtige Umstände könnte aber das Maß an Verwunderung mindern. Einerseits der Name der Gegend, wo die oben besprochenen Ereignisse alle sich abspielen, Latium nämlich. Die richtige etymologische Bedeutung ist nichts anderes als Wasserland, Sumpfland, auf Grund der Verwandtschaft mit solchen Wörtern wie lacus ,See, Lache, Sumpf“, ein Ergebnis,

${ }^{62}$ Der Fluß Ufens fließt durch volskisches Land. Die Volsker gehören zur umbrischen Sprachgruppe. Ein umbrisches $-f$ - entspricht lateinischem $-b-$, vgl. umb. alfer $=$ lat. albus. So kann umbr. $u f$ - lateinischem $u b$ - entsprechen, was ein Stamm mit der Bedeutung „fruchtbar“ ist, vgl. lat uber, uberare, ubertare. -ens ist das bekannte Suffix des Partizip Praesens Aktiv. Umbrisch Ufens kann also von einem angenommenen Verb *ub-ere = „befruchten, fruchtbar machen“ als Part. Praes. Akt. in der Bedeutung „Fruchtbarkeit gebend“ abgeleitet werden. Vgl. die Analogie der lat. Verben uberare, ubertare. Leumann, 1977, 169, 173. Adamik, 2009, 71, 81, 90, 110.

${ }^{63}$ Serv., ad Aen. VII 799. Latte1960, $189^{3}$

${ }^{64}$ Epigrammata Bobiensia 48,3 . Latte ,1960, 78

${ }^{65}$ Vell. Pat. II 19. Luc. II 424.

${ }^{66}$ Mingazzini 1938, 941 ff. Latte1960, 192 , und Anm. 4. 
das die Wissenschaft Weitgehend Béla Adamik zu verdanken hat. ${ }^{67}$ Auf der anderen Seite ist an die Bedeutung des Wortes pontifex „Wegebahner“, „Brückenbauer" und die entscheidende Rolle ihrer Träger in Rom seit frühen Zeiten zu erinnern. Dieser Name erinnert an die Überwindung einer ähnlichen Schwierigkeit der Fortbewegung, wie sie die Anfangsszene der Camilla-Geschichte bildet. Die Verbindung junger Frauen mit einer so gearteten Umwelt erscheint letzten Endes gut verständlich, da auch die griechischen Amazonen schon, wie bereits erwähnt, neben einem Fluß leben, dem Thermodon, ${ }^{68} \mathrm{Flu}$ einer fruchtbaren Ebene. Übereinstimmungen, die eine nähere Betrachtung des Verhältnisses Camillas und ihrer Verwandtengestalten zum Vorbild der griechischen Amazonen hier nahe legen.

$\mathrm{D}$

Griechische Vorbilder

Verschüttetes muß ... gehoben werden... F. Altheim, Italien und Rom, 14.

Es kann darüber kein Zweifel bestehen, daß Vergil bestrebt war, sein Epos nach dem Vorbild der griechischen Epik mit amazonenartigen Gestalten zu schmücken wie dies in der bisherigen Forschung bereits intensiv herausgearbeitet werden konnte: Insbesondere Harpalyce, Hippolyte und Penthesilea dienten ihm als Vorbild, die alle drei in seinem Gedicht auch namentlich erwähnt werden. ${ }^{69}$ Es wäre jedoch ein Fehler, wie es mitunter geschieht, für sicher zu halten, daß die Existenz griechischer Vorbilder die einzige Veranlassung, für Vergil zur Einführung kämpfender Frauen in sein Epos war oder sich nur auf die übereinstimmenden Punkte zwischen Vorbild und Neuschöpfung zu beschränken. Was das erstere betrifft, ist es eine Tatsache, daß es in Mittelitalien Gräberfunde mit bewaffneten Frauen gibt ${ }^{70}$ und es nicht ausgeschlossen werden kann, daß Vergil von solchen Existenzen irgendwelche Kunde, wie dunkel auch immer, gehabt hat. Was das zweite betrifft, sollten wesentliche Unterschiede zwischen den genannten griechischen kämpfenden Frauen und Vergils Gestalten nicht übersehen werden, in denen sich wichtige Aussagen des Dichters verbergen. Zum Beispiel hat Harpalyke einen räuberischen Charakter, wie schon

\footnotetext{
${ }^{67}$ Adamik 2003, $189 \mathrm{ff}$.

${ }^{68}$ S. oben Anm. 24.

${ }^{69}$ Harpalyce: Aen. I 314 ff. Penthesilea: I 490 ff.: Ducit Amazonum ... agmina / Penthesile furens ... / ... Audetque viris concurrere virgo. XI 660 ff.: bellantur Amazones ... circum Hippolyten ... se ... Penthesilea ... refert.

${ }^{70}$ Köves-Zulauf 1988 (1978) 299 (431).
} 
der durchsichtige Name „Räuberische Wölfin“ es besagt, beraubt regelmäßig die Bauern, gegen die sie ihre Kämpfe auszufechten pflegt. ${ }^{71}$ Dieses Wölfische ist im grundsätzlichen Gegensatz zu dem „myrtisch“ pastoralen Welt Camillas in der Wildnis, die sie anders als Harpalyke, nicht regelmäßig verlässt, eine Welt, die gerade gegen Wölfe durch die Hirten verteidigt werden muß. Camillas Mörder ist es, der mit einem Wolf verglichen wird, der einen Hirten getötet hat: er ist wie ein occiso pastore lupus (XI 811). Auf der anderen Seite ist Penthesilea im Tode so schön, daß ihr Mörder, Achill, sich in sie verliebt. ${ }^{72}$ Demgegenüber wird Camillas Schönheit im Tod vernichtet, sie ist eine „vom düsteren Tod entstellte" (tristi mulcata morte Camilla - XI 839). ${ }^{73}$

Solche Unterschiede zwischen griechischem Vorbild und vergilischer Nachbildung - besser gesagt vielleicht: Neuschöpfung - festzustellen und zu deuten scheint ein noch nicht ganz abgeerntetes Feld zu sein. Auch die Münze Vergils hat zwei Seiten, hinten die schöne Erzählung, aus welcher Quelle auch immer, vorne aber die Zahl als die den Wert bestimmende Aussage. Und diese Aussage erfolgt in römischer Zeichensprache, für Römer. Latiums kämpfende Frauen sind Italides (XI 657), tragen Namen aus der einheimischen Tradition, die griechischen Amazonen sind nur Vergleichgrößen Quales ... Amazones (XI 659660), wie lehrreich mögen sie auch immer sein. Sie haben nur Geltung, sofern sie in die Welt Latiums integriert sind, wie die trojanischen Ankömmlinge in Italien integriert werden, in Zukunft lateinisch sprechen, Latiner heißen werden (XII 820-841). Die erörterte systematische Bindung von virgines und viragines an Gewässer des Wasserlands Latium ist auch ein wichtiges Moment dieser "Italitas" der Aeneis.

Vor diesem Hintergrund ist auch die Frage nach der originären Natur der Camilla-Gestalt in adäquater Weise zu stellen. Es dürfte einerseits sicher sein, daß sie letzten Endes eine Schöpfung poetischer Gestaltungskraft Vergils ist, andrerseits aber nicht restlos Produkt seiner dichterischen Phantasie. Er hat zum erheblichen Teil vorgefundenen Stoff verarbeitet, Gestalten der griechischen Epik, aber auch die Paluda des Ennius in der Gestalt Iuturnas in seinem Werk nachgebildet. Meine Annahme ist, daß er dabei auch die weiteren in der älteren römischen Literatur „,versunkenen“ Figuren nach Paluda-Art, von Satura bis Marica im Kopf haben konnte und auf diese Weise indirekt auch historische

\footnotetext{
${ }^{71}$ Serv., ad Aen. I 31. Hyg., fab. 193. Knaack 1894, 526 ff.

${ }^{72}$ Quint. Smyrn. I 622 ff., 660 ff., 671 ff. Penthesilea ist im Tode schön wie die Göttin Artemis. Die Göttin Aphrodite hat sie zu einem Wunder an Schönheit im Tode gemacht. Prop. III $11,15 \mathrm{f}$.

${ }^{73}$ S. oben S. 19.
} 
Realitäten des archaischen Italien. ${ }^{74} \mathrm{Ob}$ an diesen Gestalten auch folklorartige mündliche Erzählungen hingen, wie rudimentär auch immer, und solche Vergil bekannt waren, ist eine nicht zu klärende Frage. Angesichts der Wichtigkeit und Außerordentlichkeit dieser Figuren ist es vielleicht nicht ganz unwahrscheinlich. ${ }^{75}$ Auf diese Weise kann Camilla zwar keine persönliche, wohl aber eine typologische Realität zuerkannt werden ${ }^{76}$. Auch sie ist wie das ganze Altitalien Vergils eine Mischung aus Realität und dichterischer Phantasie

E

\section{Decus Italiae - Dedecus Mundi}

Camillas horrenda $=$ „furchterregender" Charakter ergibt sich für Turnus aus zwei Gründen: einerseits schon weil sie Waffen trägt, andrerseits aber weil sie dies als Frau tut, amazonenhaft ist. ${ }^{77}$ Waffen als potentielle Mittel zum Töten können per se Furcht erzeugen, bewaffnete Frauen müssen es, wenn sie der herrschenden Gesellschaftsordnung widersprechen, diese damit gefährden. Furcht verwandelt sich aber in Lob, wenn man bedenkt, daß diese zerstörerische Potenz gegen die Feinde und damit für den eigenen Schutz verwendet werden soll. In diesem Sinne wird Camilla für Zierde des eigenen Landes, Italiens erklärt, ein einmalig formuliertes Lob in der römischen Literatur, ob für Männer oder Frauen. Und eine einmalige Wendung in der Aeneis für Personen, die vorher horrendae genannt wurden. ${ }^{78}$.

Insofern kann als Antwort auf die am Anfang des Vortrags gestellte Frage nach der Natur des Widerspruchs zwischen horrenda virgo und decus festgestellt werden, daß dies nur ein potentieller Widerspruch ist, kein aktueller, wirklicher. In ihm verbirgt sich eine andere beachtenswerte Erkenntnis, die Erkenntnis nämlich, daß eine Waffen tragende Frau wie Camilla von der latinischen Gesellschaft ihrer Zeit, nach der Darstellung Vergils akzeptiert werden konnte. Das ist die Implikation ihres Gelobt-Werdens. Ihr Kampf für Latium als Repräsentantin der amor patriae kann sogar zum Vorbild auch für die gewöhnlichen Frauen werden. ${ }^{79}$ Ein echter Widerspruch zu ihrer Charakterisierung als decus Italiae ist aber in anderer Richtung zu finden, und diesmal ist der Widerspruch absolut und echt. Er ist im Urteil ihres Mörders Arruns enthalten, der

\footnotetext{
${ }^{74}$ Arrigoni 1982, 103: „il peso della tradizione letteraria greca ... non è completamente responsabile della creazione di Camilla."

${ }^{75}$ Köves-Zulauf 1972, 226, Anm. 347. Cristobal, 1988-1989, 45.

${ }^{76}$ Arrigoni 1982, 72: „,non credo che Virgilio abbia totalmente inventato la sua Camilla“.

${ }^{77}$ XI 498 ff. insbes. das Nacheinander in 507: ad haec oculos horrenda in virgine fixus.

${ }^{78}$ S. oben Anm. 2. Aen, III 658. III 679. IV 181. VI 10. VI 298. VII 323. IX 521.

${ }^{79}$ Aen. XI $891 \mathrm{ff}$.
} 
der Wertung des Turnus als decus das Urteil dedecus entgegensetzt und Camillas Wesen im Übereinstimmung mit dedecus weiter als dira pestis = „grauenvolles Scheusal“ verdeutlicht und sie mit dieser Begründung tötet ${ }^{80}$. Dies entspricht der offiziellen Wertung des Roms späterer historischer Zeiten, das jedes androgyne Sein als Gefährdung der Weltordnung, als prodigium wertete und von Amts wegen beseitigen lie $\beta{ }^{81}$ Ein solcher Widerspruch zwischen der Wertung Camillas als decus oder dedecus ist in mehrfacher Hinsicht bemerkenswert. Dadurch wird erstens die Existentberechtigung von Waffen tragenden Frauen in der Aeneis problematisiert, was sich zweitens als eine typisch römische Fragestellung erweist. Drittens aber werden die zwei Wertungen indirekt auch zeitlich eingeordnet. Akzeptiert, ja sogar auf ein Piedestal gestellt wird nämlich eine solche Existenz durch Vertreter einer im Vergehen begriffenen Welt, die gegen das Fatum d.h. gegen die Zukunft sich stellen, während der Mörder Camillas das Fatum ${ }^{82}$ d.h. die Zukunft vertritt, die für Vergil im augusteischen Rom ihre Endgestalt gewann. Auch Iuturnas kriegerische Tätigkeit endet parallel mit der Existenz des Repräsentanten der Welt der Vergangenheit, Turnus. Nach der Darstellung Vergils existierten also in grauer Urzeit in Italien nicht nur bewaffnete Frauen, sondern wurde diese Existenz auch positiv gesehen, zwei Tatbestände, die eng zusammenhängen.

Es ist nun interessant festzustellen, daß ein ähnlicher Unterschied hinsichtlich eines so wichtigen Lebensumstandes bewaffneter Frauen vorhanden zu sein scheint, wie das Verhältnis zu Gewässern, insbesondere zu Sümpfen ihrer Umwelt. Daß Sümpfe in Zeiten Acker bauenden, Straßen anlegenden Leben als hinderlich gelten, und deswegen überwiegend negativ gesehen werden, gilt auch für die Geschichte des antiken Rom. Für Cicero waren die pomptinischen Sümpfe „ein weder angenehmes, noch gesundes Gebiet“ ${ }^{83}$ für auf einer Reise in der Nähe des Sumpfgebiets übernachtenden Horatius, wo auch Camillas anzunehmender Weg nach Vergil einst vorbeiführen musste, ein Ort "böser Mücken und Schlaf vertreibender Sumpffrösche. ${ }^{\text {“84 }}$ Auf der anderen Seite gibt

${ }^{80}$ Aen. XI 789. 791

${ }^{81}$ Köves-Zulauf 1988 (1963), 84 (33) Anm. 24

${ }^{82}$ Er ist fatis debitus XI 759, ein doppelsinniger Zustand. Es bedeutet einerseits: „dem Schicksal verpflichtet“, andrerseits „,dem Schicksal verfallen“. Einerseits ist sein Schicksal, daß er Camilla töten muß, weil ihre Existenz gegen die Weltordnung verstößt: Camilla fatis urgetur acerbis ,vom bitteren Schicksal bedrängt wird“ XI 587. Andrerseits verlangt das Schicksal, daß er sterben muß, weil er seine Schicksalspflicht, die Tötung Camillas, nur durch einen Verstoß gegen ein göttliches Gesetz erfüllen kann: quicumque sacrum violarit vulnere corpus ... morte luet merita: XI 591. Köves-Zulauf 1988 (1978), 269(198) ff.

${ }^{83}$ De Orat. II 290.

${ }^{84}$ Sat. I 5,14 
es Spuren einstiger vorwiegend positiver Wertungen solcher Sumpfgebiete. ${ }^{85}$ Man schrieb ihnen große Fruchtbarkeit zu, Wasser war sozusagen der Dünger solcher Zeiten. ${ }^{86}$ Denn eine Capra, Ziege, ist ein weit verbreitetes Symbol für Fruchtbarkeit, Satura bedeutet „satt", "reichhaltig“, „fruchtbar". In dieselbe Richtung weist aber auch Name und Schicksal der paludes Pomptinae, eines Sumpfgebietes, das die auf den Kriegsschauplatz ziehende Camilla berühren musste, und dessen Teil die Saturae palus war. Der Name Pomptinae leitet sich vom Wort pomum „Obst“ ab. Zwei Traditionen weisen außerdem auf die große Reichhaltigkeit dieses Sumpfgebietes hin. Der letzte römische König soll aus der Beute, die er durch Eroberung der Stadt Pometia „Obststadt“, die in diesem Sumpfgebiet lag, machte, den Bau des Kapitols finanziert haben ${ }^{87}$ Nach lokaler Tradition blühten im Sumpf ehedem 24 Städte, die als spurlos verschwunden galten. ${ }^{88}$ Aber „Fruchtbarkeit Gebend“ ist auch die etymologische Bedeutung des Namens des Flusses Ufens, der durch die paludes Pomptinae fließt und in unmittelbarer Nähe Camillas in der Heerschau erwähnt wird. ${ }^{89}$ Aber auch das Wasser Iuturnas, das zugleich in Form von Quelle, Teich, Sumpf da war, ${ }^{90}$ galt als heilkräftig ${ }^{91}$ und äußerst heilig ${ }^{92}$.

Andrerseits waren sie Sümpfe von Natur aus geschützte Gebiete, Sumpfwasser eine Art naturgegebene Schutzmauer. ${ }^{93}$ Diesbezüglich kann an die alte falsche - Etymologie von Latium erinnert werden, die das Wort von latere, „sich verstecken“, ableitet. ${ }^{94}$ Als Urbeispiel für solches Verstecken berichtet gerade Vergil das latere des Gottes Kronos = Saturnus auf dem Gebiet des späteren Rom. ${ }^{95}$ Bei näherem Bedenken der Einzelheiten, was von diesem Tun des Saturnus berichtet wird, und was wir von der geographischen Beschaffenheit

\footnotetext{
${ }^{85} \mathrm{Zu}$ der großen Bedeutung von Sümpfen und ähnlichen Wassergebilden im alten Rom s. Borca 1995, $55 \mathrm{ff}$.

${ }^{86}$ Sumpfwasser, Schlamm konnte noch in klassischen Zeiten diese Rolle spielen: Verg., G. I 113-116: quique paludis / collectum umorem bibula deducit harena /... amnis abundans / ... obducto tenet omnia limo $=$,und wer das gesammelte $\mathrm{Na}$ des Sumpfes durch den durstigen Sand führt ... der überfließende Strom hält alles unter einer Schlammdecke.“

${ }^{87}$ Liv. I 53,2-3. Cic., Rep. II 44 etc. S. Der Kleine Pauly s.v. Suessa Pometia 8.

${ }^{88}$ Plin., Nat. VII 68-69. Köves-Zulauf 1988 (1963), 81 (30) ff., 92 (41) ff.

${ }^{89}$ S. oben Anm. 62.

${ }^{90}$ Fons: Stat., Silv. IV 5,35. lacus: Ov., Fast. I 708.Val. Max. I 8,1. stagna: Ov., Fast. II 603. lympha: Varro L. V 10,71.

${ }^{91}$ Varro L. V, 10,71.

${ }^{92}$ Aus dem lacus Iuturnae wurde Wasser für alle staatlichen Opfer geholt: Serv., ad Aen. XII 139.

${ }^{93}$ Wasserburgen späterer Zeiten beruhen auf der Ausnutzung ähnlicher Geländeformationen.

${ }^{94}$ Malby 1990 , s.v.

${ }^{95}$ Aen. VIII 319-322 f.: Saturnus ... Latiumque vocari / maluit, his quoniam latuisset tutus in oris. Auch die Saturnia Iuno versteckt sich, vgl. Johnston 1977, 64 ff.
} 
des späteren Stadtgebietes wissen, kann dieses Verstecken kaum anderswo als in einem Sumpf erfolgt sein. ${ }^{96}$ Von einer solchen positiven Möglichkeit wurde gelegentlich noch in historischen Zeiten Gebrauch gemacht. Noch in der frühen Kaiserzeit galten die pomptinischen Sümpfe als Versteck für Räuber. ${ }^{97}$ Auch Marius hat in Lebensgefahr in einem Sumpf in Latium, gerade im Sumpf der Marica (!) sich versteckt, ${ }^{98}$ ebenso sein Mitkämpfer P. Sulpicius Rufus in den laurentinischen Sümpfen ${ }^{99}$. Für Phaedrus ist paludis latibula „Sumpf als Schlupfwinkel“" ein fester Begriff (I 30,91).

So stellt sich zum Schluß die Frage, was Vergil durch die Betonung der aquatischen Gebundenheit der nach seiner Vorstellung und in seiner Darstellung in den „heroisch“ zu nennenden Zeiten in Italien vorhandenen „Amazonen“ aussagen wollte. Eine komplexe, den hier gegebenen Rahmen sprengende Frage, deren meritorische Behandlung deswegen - dis volentibus - der Zukunft vorbehalten bleiben muß. Und wenn auf diese Weise meine Überlegungen hier eher nur aufhören als zu Ende geführt werden können, so dürfte dies nicht gegen die Natur der Wissenschaft sein. Denn daß Ergebnisse eigentlich nur Vorbereitungen für erneute Fragen sind, dürfte zum Schicksal wissenschaftlichen Forschens gehören. Ars non solum longa, sed numquam finita. So stehe hier als Abschluß, und zugleich als Aufforderung zum Weiterdenken wenigstens die Feststellung, daß das Thema bewaffneter Jungfrauen und ihr Platz in umweltlicher Umgebung ein charakteristisches und wichtiges vergilisches Thema war. Denn dieser Homer der Römer hat mit der Aeneis ein sehr komplexes Werk geschaffen, von hoher Qualität in aesthetischer, geschichtskundiger, ideologischer Hinsicht. Wer könnte in der Tat die ästhetische Qualität der Schilderung des Auftritts Camillas in der Heerschau ernsthaft in Frage stellen ut gerat ... pastoralem praefixa cuspide myrtum $=$,wie ... ihre Gestalt ... trägt den Myr-

\footnotetext{
${ }^{96}$ Eine andere Landschaft des zukünftigen Rom, die sich zum Sich-Verstecken anbot, war der Wald: Hier versteckte sich Lavinia und gebar den Aeneas Silvius. Dionys. Hal. I 70,1-3. Origo gentis Romae 16,1. Silvius als Beiname der albanischen Könige ist ein Hinweis auf die wichtige Rolle des Waldes in Urzeiten. Auch wenn man Saturnus sich als in einem Waldgebiet versteckt vorgestellt haben sollte, und darauf den Namen Latium zurückführte, bleibt bestehen, daß man dem Verstecken in alten Zeiten große Bedeutung zuschrieb und dazu ein Sumpf sehr geeignet war. Nicht zufällig bedeutet der Name Velabrum eines Sumpfes auf dem späteren Stadtgebiet Roms „Verstecksort“: velare + Suffix -brum. Carandini 2002, § 77, S. 143., 264 Taf. IV-X.§ 83 S. 150, § 89 S. 157 etc. Vgl. velabrum mit der Bedeutung „Schirm“ bei Ammianus Marcellinus 14,6, 25: nonnulli sub velabris umbraculorum theatralium latent.

97 Juv. III 307.

${ }^{98}$ Ov. Epist. ex P. IV 3,46. Cic., In Pis. 43. Fin. II 32,105. Vell. Pat. II 19,2 Ampelius, Liber mem. 42,2. Liv. periochae 77.

${ }^{99}$ Vell. Pat. II $19,1$.
} 
tenspeer der Hirten mit eiserner Spitze “ (VII 806 ff.) oder Iuturnas Abschiedsworte in ihrer berühmten „Klage." ${ }^{\text {"100 } O}$ quae satis ima dehiscat / terra mihi, Manisque deam demittat ad imos?" = „Ach wo täte sich tief genug die Erde mir auf und schickte (mich obwohl) Göttin tief hinab zu den Manen?"(XII 883 ff.). Auch erweist Vergil sich mit der Schilderung von bewaffneten Frauen im alten Italien, deren reales Vorhandensein archäologische Funde bestätigen, erneut als einen hervorragenden Kenner italischer Vorgeschichte wie man ihn mit Recht genannt hat. ${ }^{101}$ In diesen Rahmen ordnet sich ein des Dichters Hinweis auf die Wasserverbundenheit italischer "Jungfrauen in Waffen“ Schließlich verdient volle Anerkennung, daß er als Schöpfer eines repräsentativen Werkes des augusteischen Zeitgeistes zugleich sich fähig zeigt, die Kategorien der eigenen Zeit historisch zu relativieren und durch diese Schilderung des Andersseins früherer Zeiten Zeugnis ablegt von dem, was man echten Sinn für Geschichte nennen könnte. So spricht die Betrachtung der Schilderung von Camilla, Iuturna und Schicksalsgenossinnen erneut dafür, daß Vergil zu lesen nicht nur genußvoll, sondern auch lehrreich, ja zum Nachdenken anregend ist.

\section{Bibliographie}

Adamik 2003 = Adamik, B.: Die etymologische Herleitung des Namens Latium. In: Calboli, G. (ed.): Latina Lingua. Proceedings of the $12^{\text {th }}$ International Colloquium on Latin Linguistics, Bologna, 9-14 June 2003. Roma, 189-198.

- $2009=$ Adamik, B.: A latin nyelv története (Geschichte der lateinischen Sprache). Budapest.

Alföldi 1959 = Alföldi, A.: Hasta-Summa imperii. AJA 63, 1-25.

Altheim 1931 = Altheim, F.: Messapus. ARW 29, 23-32.

- $1956=$ Altheim, F.: Römische Religionsgeschichte I-II, 2. Auflage. Berlin.

Arragoni 1982 = Arrigoni, G.: Camilla, Amazzone e sacerdotessa di Diana. CisalpinoGoliardica.

Binder 2012 = Binder, G.: P. Vergilius Maro, Aeneis. Stuttgart.

Borca 1995 = Borca, F.: Fondazione e fango: Romolo, la palude e le radici della cultura romana. Aufidus 26, 55-68

Carandini 2002 = Carandini, A.: Die Geburt Roms. Düsseldorf-Zürich.

Cristobal 1988-89 = Cristobal, V.: Camila: Génesis, función y tradición de un personaje virgiliano. Estudios clásicos 31, 43-61.

Della Corte 1972 = Della Corte, F.: La mappa dell'Eneide. Firenze.

Dumézil, G. 1966 = La religion Romaine archaïque. Paris.

Fratantuono - Osker $2010=$ Fratantuono, L. M. - Osker, M. F.: Camilla and Cydippe: A note on Aeneid 11,581-582. QUCC 96, 111-116.

\footnotetext{
${ }^{100}$ Perkell 1997, 257-286.

${ }^{101}$ Alföldi 1959, 3. Köves-Zulauf 2013, 225 ff.
} 
Georges $1962(1913)=$ Georges, K. E.-H.: Lateinisch-Deutsches Handwörterbuch. Hannover, $1962^{11}\left(1913^{8}\right)$.

Horsfall $1988=$ Horsfall, N.: Camilla, o i limiti dell'invenzione. Athenaeum 66, 31-51.

$-2000=$ Horsfall, N.: Virgil, Aeneid 7. Leiden-Boston-Köln.

- $2003=$ Horsfall, N.: Virgil, Aeneid 11. Leiden-Boston.

Johnston 1977 = Johnston P. A.: Vergil's Conception of Saturnus. Calif. Stud. Class. Ant. $10,57-70$

Knaack 1894 = Knaack, G.: Harpalyke. $R h M$ 49, 526-531.

Köves-Zulauf 1972 = Köves-Zulauf, Th.: Reden und Schweigen, München.

— 1988 = Köves-Zulauf, Th.: Kleine Schriften. Heidelberg,

81-112: Plinius über den Untergang der Stadt Pometia (1963); 253-304: Camilla (1978).

— 2013 = Köves-Zulauf, Th.: Hasta Societatis. Lanzenkult im antiken Rom. ACD 49, 219- 241.

- 2016 = Köves-Zulauf, Th.: Iuno. Ókor (Altertum) 39-51.

KP 1979 = Der Kleine Pauly. München 1: Amazones, 292.

4: Poseidon, 1076.

5: Varro, 1133.

5: Tullius nr. 5, 997, $59 \mathrm{ff}$.

Kretschmer $1923=$ Kretschmer, P.: = Das $-n t$ Suffix, Glotta, 14 .

Latte 1960 = Latte K.: Römische Religionsgeschichte. München

Leumann 1977 = Leumann, M.: Lateinische Laut- und Formenlehre. Handbuch II.2.1. München.

Malthy $1990=$ Maltby, R.: A Lexicon of Ancient Latin Etymologies. In: Arca Classical and Medieval Texts, Papers and Monographs, 25.

Mingazzini 1938 = Mingazzini, P.: Il santuario della dea Marica alle foci del Gargigliano. In: Monumenti Antichi p. dai Lincei 32, 2, $94 \mathrm{ff}$.

Perkell 1997 = Perkell, Ch.: The Lament of Juturna: Pathos and Interpretation in the Aeneid. TAPhA 127, 257, 286.

Tarleton 1989 = Tarleton, N.: Pastoralem praefixa cuspide myrtum (Aeneid 7.817). Classical Quarterly 39, 267-8.

Torrão 1993 = Torrão, J. M. N.: Camila, a virgem guerreira. Humanitas 45, 113-136.

(ISSN $0418-453 X)$ 